\title{
Holistic Understanding for Design - Theory of Technical Systems
}

\author{
W. Ernst Eder \\ Royal Military College of Canada (retired) \\ eder-e@kos.net
}

\begin{abstract}
Improvements at local to global levels needs holistic understanding of the processes and actors (operators). The theory of technical systems provides a model for a holistic understanding, and a conceptual basis for qualitative thinking. The theory describes a transformation system, in an environment. Any artificial change (transformation) can be modeled in this way. The environment includes local influences and effects acting on the system and its process, and a general environment (regional, national and global) that covers physical, chemical, societal, economic, cultural, political, ideological, geographic and all other influences, with a link to other areas of study.

Technical systems (as main operator) experience a typical life cycle. This leads to consideration of supply networks, globalization, financing, impacts on the environment, and other concepts. The process, and all operators, exhibit typical classes of properties. Each process and operator consists of elements and relationships that form structures of several useful kinds. Anticipating a future involves establishing the requirements, including those that arise from the producing organization. The available technological and scientific information influences the development of individual sorts of transformation system, and enables and limits the changes in culture that can be implemented.

A rational methodology for designing newer transformation systems can be proposed. This systematic approach to designing allows use of other design methods, including intuitive working.
\end{abstract}

\section{Introduction}

A major difference between science and engineering has been stated as:

- scientific activity tries to extend the boundaries of knowledge, preferably for a short boundary length of a specific knowledge region at a time, with reference to its existing knowledge base;
- engineering tries to create working systems, mostly within the boundaries and complex interactions of many knowledge and information regions.

The processes and actors (operators) for any engineering improvements at local, regional, national and global levels need to be understood in a holistic way. Any change at one level will inevitably influence other levels, the viewpoints are hierarchical - a system is a member of a higher-order system, and can usually be divided into sub-systems. Changes will need to be planned with care, and will have some foreseen consequences, and some unexpected results. Engineering education should thus aim to instil that complex interactive holistic understanding.

The theory of technical systems (TTS) $[1,2,3]$ provides a comprehensive model from which this holistic understanding can be obtained, and presents a conceptual basis for qualitative thinking, especially useful in engineering education. The following sections present a brief and incomplete outline, with comments on context, relevance and applicability.

\section{Theory of Technical Systems}

According to ISO 9000:2005 [4], a 'product' is defined as a 'result of any process'. A product is the operand in state $\mathrm{Od} 2$ at the end of a transformation process (TrfP), and is classified as:

(a) hardware: a tangible, material object, with countable quantity;

(b) software: information, and an intangible object (an insurance policy, a computer program, etc.);

(c) service: the intangible result of an activity performed at the interface between a supplier and a customer; this includes provision of electricity, water, fuel, transportation, garbage removal, policing, wholesale and retail, advertising, delivery of information, providing ambience, etc.; and

(d) processed material: a solid, liquid or gaseous (bulk) material that can be measured in units of volume, mass, energy, etc., e.g. plastics, fuel, grease, coolant. 
To (a): a range of products was described in [2] p.1012 , and in [3] p. 336-337. Those tangible products that have a substantial engineering content are called technical system, TS. To (c): this also includes an activity that results in a change in a tangible or intangible operand, i.e. a process product, a transformation process, TrfP. Further to (c): 'supplier' and 'customer' are to be understood in a very wide sense - an employee on the manufacturing line is a 'customer' for the information delivered by the design engineering department. Also to (c) and (d): energy is only listed here as manifested by its carriers. This definition is more explicit than the usual designation of 'goods and services'.

The theory of technical systems describes in general terms and interconnected models the common traits of all process products and tangible products that have a substantial engineering content. The basic model shows a transformation system (TrfS), within a general environment, figure 1. This TrfS consists typically of a transformation process (TrfP) which transforms an operand (Od) in structure, form, space and time, driven by effects delivered via a technology (Tg) by five (classes of) operators: human systems (HuS), technical systems (TS), active and reactive environment (AEnv), information systems (IS), and management systems (MgtS). The TrfP is external to the operators, the operators can act and/or work even if no operand is present.

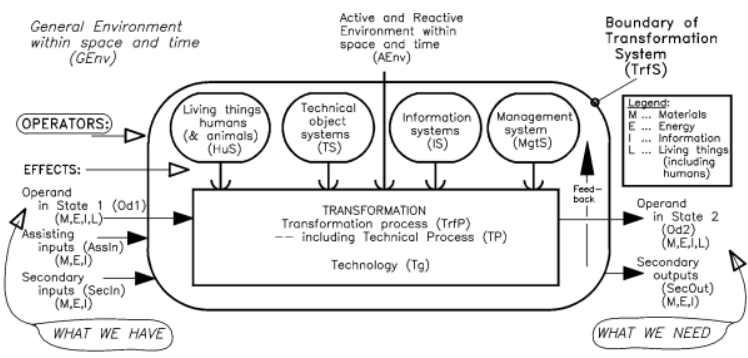

Figure 1. General Model of a Transformation System (TrfS) [3]

Any artificial change (transformation) can be modeled in this way. Examples are: (1) hardening a steel workpiece by heating and quenching, or inserting a heart by-pass - change of structure; (2) machining a workpiece, or applying a prosthetic hand - change of form; (3) transporting goods or people from one place to another - change of location; and (4) storing goods, or going to sleep - change in time.

The transformation system according to this model is a Product Service System - but this term arose many years after Hubka first proposed the TrfS model in 1967 [5]. The main purpose of any TS (as tangible product) is usually to perform a transformation process, TrfP. You look for something with which to calculate with numbers and display results - an abacus, a pocket-calculator, or a computer.

The environment includes local influences acting on or reacting to the system, and effects acting on or reacting to the operand in its process. The general environment (regional, national and global) covers physical, chemical, societal, economic, cultural, political, ideological, geographic and all other influences indirectly acting on or reacting to the TrfS. A link can thus be made to the humanities, politics, and other areas of study, which can help to make engineering into 'liberal' education.

The operand input to a TrfP, Od1, includes all influences on the environment caused by previously reaching that input state. The operand output, Od2, is intended to be improved from its input state, Od1 conservation of material and energy is observed, information may be added or deleted. The operand output, Od2, is usually accompanied by secondary outputs, which may be useful for other purposes, or add to waste and pollution - a direct link to environmental impact concerns. This demonstrates that TTS is useful in engineering education as an explanatory system for the context of design engineering, and for interactions of the engineering sciences.

\section{Technical Systems - Life Cycle}

The technical system TS(s), as subject and main operator of interest, is an artificial, human-made tangible product. Its typical life cycle consists of seven (classes of) transformation system, with relevant processes of: $\mathrm{LC} 1$ - product planning of the $\operatorname{TrfP}(\mathrm{s})$ and the TS(s); LC2 - designing of the TrfP(s) and/or the TS(s); LC3 - manufacturing planning, technological and organizational preparation for the subsequent life-cycle phases; LC4 - manufacturing and assembly of the TS(s); LC5 - distribution, sale, delivery of the TS(s); LC6 - operation of the TS(s) and performance of the $\operatorname{TrfP}(\mathrm{s})$; LC6a - servicing, maintaining, repairing the $\mathrm{TS}(\mathrm{s})$; and LC7 liquidation of the TS(s), recycling. Each life-cycle transformation systems is comprised of other transformation systems - a hierarchical network.

This leads to consideration of various concepts. Supply networks are needed to deliver raw materials and OEM (original equipment manufacturers) goods. OEM goods appear mainly as machine elements [6], including electronic, hydraulic and others not usually found in mechanical products. The influences of globalization, suppliers world-wide, reducing local economies, and financing can be shown. Life-cycle 
processes are influenced by their environments, but also have impacts locally and globally. The context to design engineering should be obvious.

\section{Properties, Behaviour}

The process, and all operators in their existing 'as is' state, exhibit typical classes of properties. Each process and TS-operator consists of elements and relationships that form structures of several kinds that have been found useful for conceptual design engineering. The primary classes of properties for a transformation process, TrfP, are shown in figure 2, and for a technical system, TS, in figure 3. All actual properties can be allocated into these classes - no other classes are needed. In each case, the observable properties can generally be deduced, observed, detected, assessed and/or measured by a lay person from the appearance and literature of the product.

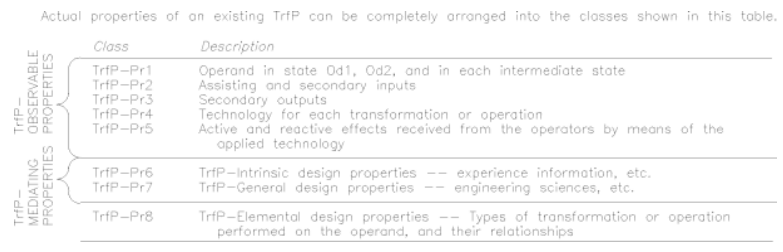

Figure 2. Primary Classes of Properties of a Transformation Process (TrfP)

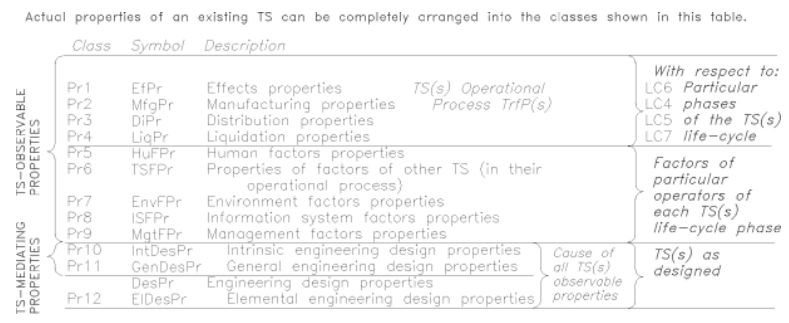

Figure 3. Primary Classes of Properties of a Technical System (TS)

The classes of TS-mediating properties comprise (a) the TS-intrinsic design properties of technical information and accumulated experience, including action locations, technological principles, mode of action, available effects, principles of form-giving, and many others, is often organization-specific, and is of little interest to science; and (b) the TS-general design properties which relate to the applicable engineering sciences. The TS-elemental properties are directly controlled by engineering designers, and some of these are manifested in some TS-observable and TSintrinsic design properties.
Elements of a transformation process are also hierarchically transformation processes, down to those that are no longer usefully sub-divided - operations.

Among the TS-elemental properties are the various possible TS-structures, see figure 5.4, [1] pages 67-69, figure 7-3, [2] pages 106-107, and [3] pages 294-295, figure 6.3: Elements of technical systems are TSinternal and cross-boundary functions, organs, and constructional parts: (1) TS-Function structure using TS-internal and cross-boundary functions as elements - functions describe (usually in words) the capability for TS-internal and cross-boundary action; (2) TSorgan structure using TS-internal and cross-boundary organs as elements - organs are sites where interactions between constructional parts take place; and (3) TS-constructional structure using TS-internal and boundary constructional parts. The constructional parts may have (classes of) level of abstraction of modeling, form, size, material (with mechanical, electrical, chemical, thermal, optical, technological and other properties), size deviation, surface quality (texture, reflectivity, color, ...), etc.

Structure (of a TrfP and/or a TS) determines behaviour, but behaviour allows several possible structures that can provide that behaviour. This allows the essence of design, a search for alternatives. The TrfP/TS-intrinsic design properties and requirements, and the TRFP/TS-general design properties and requirements provide the links between structure and behaviour - especially the mode of action [7].

Technical systems are also hierarchical. The theory of technical systems has identified four typical levels of complexity: (Level I) constructional parts are mainly simple parts that are not normally capable of further disassembly, but that can usually only be sub-divided by destroying the part - bolt, shaft, spring, resistor; (Level II) sub-assemblies of the parts, as intermediate stages of assembly; and assembly groups, modules of different complexity - gear box, shaft coupling, readwrite head; (Level III) complete assemblies (TS) that can perform a task - lathe, crane, amplifier; (Level IV) plant - oil refinery, commercial kitchen. Any TS of higher complexity consists of items of lower complexity. Even a single constructional part is regarded as a TS. The boundaries between these four levels are fuzzy. An OEM may regard a VLSI computer chip as a constructional part of complexity level I (e.g. Dell), yet the manufacturer of that chip may regard it as complexity level III (e.g. Intel). A TS of higher complexity inherits all the properties of its constituent TS of lower complexity. That TS of higher complexity also exhibits additional properties that arise from synergy among the constituent TS of lower complexity. The complexity of a TrfP is related to the complexity of the driving TS(s). 
TS-behavior is one of the important properties of a TS, and is defined as the succession of states that a TS assumes in response to its TS-internal or external stimuli. The TS-states are defined by the mixture of properties, and/or their manifestation and/or values at any one time. The states of properties exist and change under various TS-operating states, the 'duty cycle' of the TS: (a) at rest, no operation; (b) during start-up; (c) during normal operation - idling, full-power and partload, overload, etc., for self-acting operation (automatic), or running and ready to be operated by another operator, e.g. human or another TS; (d) during shut-down, ending an operational state and returning to 'at rest' conditions; (e) in fault conditions - (e1) internal faults - overload, safe trip-out, breakage or equivalent, and (e2) external faults - damage, wrecking, etc.; (f) during maintenance, repair, testing, etc.; (g) at 'life ended'; (h) any other states. TSbehavior may be predictable (and therefore causal and analyzable with the help of the engineering sciences), and/or random, and may change with time.

\section{TS-Inputs, Outputs, Effects}

In order to fulfill its purpose, to drive a TrfP, the TS(s) must receive expected (and assisting and secondary) inputs consisting of materials, information and energy $(\mathrm{M}, \mathrm{E}, \mathrm{I})$. The TS(s) in its TS-internal processes converts the M,E,I into its expected (and secondary) outputs, using its mode of action. The most significant output of a TS is its effects $(M, E, I)$, deliverable via the technology to cause change in the operand. Secondary inputs can result in disturbances of the expected behavior, secondary outputs may be useful for other purposes, or add to waste and pollution. The modes of action may include mechanical, hydraulic, pneumatic, chemical, electronic/analog, electronic/digital, electrical, nuclear, biomedical, and others, singly or in any hybrid combination. The contexts and complex interactions of the individual engineering sciences are therefore an important element of engineering education that has often been neglected. TS thus include active or reactive hybrid and high-tech devices, in a static and/or dynamic mode. The transformation can only be achieved if the TS is operational (capable of operating or being operated, fulfilling its TS-internal and cross-boundary functions) and delivers the desired effects, via the technology, to the operand of the TrfP.

\section{Development in Time}

In the past, TS were developed and adapted to local situations in slow evolution by 'directed trial and error correction', by craftsmen using their accumulated experience. An example is the range of horse-drawn carts (e.g. in England) that vary according to the geographic region and topography. Scientific research on how the TS perform their tasks usually followed much later, e.g. steam engines - that sequence has recently reversed, some scientific research now leads to some new products.

As cultural needs progress, and technological and scientific information increases, many products are developed to a better state - which in turn produces other cultural needs and more technological and scientific information. The level of available technological and scientific information influences the development of each individual sort of technical and transformation system, and thus enables and limits the cultural changes that can be implemented - explaining the development of civilizations over centuries. Such developments in time can be traced, for instance the generations of personal cars.

\section{Requirements}

Anticipating a future TrfS involves establishing the requirements for the future conditions, including those that arise from the producing organization. Requirements are closely related to properties, but their scope and information content are different. Requirements can typically set limits. Properties show the actual achieved values (preferably within the required limits), but do not allow conclusions about the initiating limits.

The two states of TrfP and TS can be illustrated, (1) existing, 'as is', with properties measurable, assessable or latent, e.g. a 'diameter of $15.9836 \mathrm{~mm}$ ', and (2) future, 'as should be', with only requirements to demand or limit the properties, e.g. 'a diameter requirement of $16 \mathrm{~mm}$ ' with tolerance grade ' $\mathrm{h} 7$ ', and heuristics to guide designers to establish and select the properties. The 'as is' state precludes finding the 'as should be' requirements - e.g. if the diameter 'as is' conforms to the requirement, the tolerance may have been 'h7', 'h8' or 'h11'. The 'as is' $\operatorname{TrfP}(\mathrm{s})$ and TS(s) cannot carry information about any alternatives that were considered during life-cycle phases LC1 - LC3. It also cannot carry information about any requirements or constraints from the life-cycle phases - the $\operatorname{TrfP}(\mathrm{s})$ and $\operatorname{TS}(\mathrm{s})$ remain within the constraints. Consequently, the primary classes of requirements are shown in figure 4 . Class $\mathrm{Rq} 1$ contains requirements for the organization, related to TS-life cycle phases LC1 - LC3. Class Rq2 contains requirements for the transformation process, analogous to figure 2 , classes Trf-Pr1 - TrfP-Pr5. Classes Rq3 - Rq11 contain 
requirements for the technical system, analogous to figure 3, classes Pr1 - Pr9. Classes Rq12 - Rq14 contain any requirements from both Trf-Pr6 - TrfPPr8 and Pr10 - Pr12.

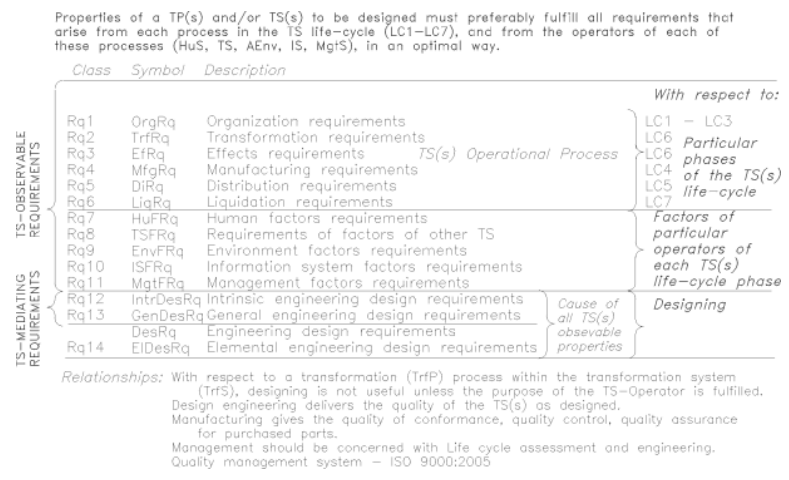

Figure 4. Primary Classes of Requirements for a Transformation System (TrfS) [8]

\section{Theory of Design Processes, and Design Method}

The theory of design engineering as proposed by Hubka [1,2,3] follows from the general model of a transformation system, figure 1 . Initially, the design process is divided into five administrative phases: product planning, task defining, conceptualizing, embodying/laying out, and detailing. At the second hierarchical level, the stages and steps of a novel design process are summarized as:

- task defining:

(P1) establish a design specification for the future system, a list of requirements, see section 7 ;

- conceptualizing:

(P2) establish the desirable and required output (operand in state $\mathrm{Od} 2$ ) of the $\operatorname{TrfP}(\mathrm{s})$;

(P3) establish a suitable transformation process, $\operatorname{TrfP}(\mathrm{s})$; and (if not stated as a requirement) establish a suitable input (operand in state Od1);

(P4) decide which of the operations in the transformation process will be performed by TS (and which by other operators);

(P5) establish which TS(s) (or parts of them) need to be designed;

(P6) establish a technology (structure, with alternatives) for that transformation operation, and therefore the effects (as outputs) needed from the technical system;

(P7) establish what capabilities the TS(s) needs (internal and cross-boundary functions, alternatives);

(P8) establish what organs (function-carriers in principle and their structure, with alternatives) can perform the functions. These organs can be found in the machine elements, as revised [6];

- embodying/laying out, and detailing:

(P9) establish with what constructional parts (in sketch-outline, in rough layout, in dimensionaldefinitive layout, then in detail and assembly drawings, with alternatives) are needed.

Redesign can be accomplished by:

(Pa) establishing a design specification for the revised system (step P1);

$(\mathrm{Pb})$ analyzing the existing system into its organs and (if needed) its functions (reversing steps (P8) and (P7) of the novel procedure);

(Pc) then following the last one or two parts of the procedure listed above for a novel system.

Superimposed on the set of design operations, at the next lower level of the hierarchy, is a cycle of basic operations of problem solving, which consists of: OpH3.1 - State the problem; Op-H3.2 - Search for solutions; Op-H3.3 - Evaluate, decide; Op-H3.4 Communicate solution. These are supported by auxiliary operations of: Op-H3.5 - Prepare information; Op-H3.6 - Check, verify, reflect; OpH3.7 - Represent. The last three were recognized by Hubka, but are rarely found in other schemes.

Only those parts of this engineering design process that are thought to be useful are employed. This systematic approach to designing and synthesizing allows use of any other design method, including intuitive and opportunistic working. The need for such a theory-based systematic design process was discussed in [9]. Such an 'idealized' procedure cannot be accomplished in a linear fashion, iterative working is essential, using the model of problem solving [7].

Because TS are hierarchical, any proposed product can be sub-divided, and each sub-division treated as a design problem in its own right. The TS-internal and cross-boundary functions in higher TS can then acts as TrfP in designing the lower TS.

The application and productivity of this design method based on the Theory of Technical Systems $[1,2,3]$ has been verified in several industrial projects from the University of West Bohemia, e.g. [10]. It has also been demonstrated in several case studies $[3,11,12]$.

\section{Closure}

The context of the theory of technical systems $[1,2,3]$ shows clearly why engineers should develop a wide and holistic outlook and insight. Engineering is not merely 'applied science' - science can be applied for analysis, and as heuristic for synthesis. Nevertheless, engineering requires a wider collection of information, skills and abilities. 
Engineering provides the tools, and is one of the basic building blocks (interacting with all others) for influencing the development of any culture. TTS also demonstrates clearly why in engineering education a narrow emphasis on the analytical engineering sciences is necessary, but by no means sufficient to produce a well-rounded and functioning engineer.

\section{References}

[1] Hubka, V. and Eder, W.E. (1988) Theory of Technical Systems (New York: Springer-Verlag

[2] Hubka, V. and Eder, W.E. (1996) Design Science: Introduction to the Needs, Scope and Organization of Engineering Design Knowledge, London: Springer-Verlag http://deseng.ryerson.ca/DesignScience/

[3] Eder, W.E. and Hosnedl, S. (2008) Design Engineering: A Manual for Enhanced Creativity, Boca Raton: CRC-Press

[4] - ISO 9000:2005 Quality Management Systems - Fundamentals and Vocabulary, Geneva: ISO, 2005 http://www.iso.ch

[5] Hubka, V., 'Der grundlegende Algorithmus für die Lösung von Konstruktionsaufgaben' (Fundamental Algorithm for the Solution of Design Problems) in XII. Internationales Wissenschaftliches Kolloquium der Technischen Hochschule Ilmenau, Heft 12 Sektion L Konstruktion, Ilmenau: T.H.I., 1967, p. 69-74

[6] Eder, W.E. (2005) 'Machine Elements Revision and Outlook for Design Education', in Proc. Second CDEN International Conference, University of Calgary, Alberta, 1819 July 2005 at Kananaskis Resort, paper 10006 on CD-ROM
[7] Eder, W.E., (2009) 'Analysis, Synthesis and Problem Solving in Design Engineering', in Proc. International Conference on Engineering Design, ICED’09, 24 - 27 August 2009, Stanford University, Stanford, CA, USA (in review)

[8] Eder, W.E. (2009) 'Properties, Requirements and Problem Solving', in in Proc. International Conference on Engineering Design, ICED'09, 24 - 27 August 2009, Stanford University, Stanford, CA, USA (in review)

[9] Eder, W.E. (2008) 'Design Engineering - Needs for a Systematic Design Process', in Proc. 10 ${ }^{\text {th }}$ Engineering and Product Design Education Conference 2008, 4-5 Sept 2008, Universitat Politecnica de Catalunya, Barcelona (Institution of Engineering Designers (UK) and The Design Society)

[10] Hosnedl, S., Srp, Z. and Dvorak, J. (2008) 'Cooperation of Engineering and Industrial Designers on Industrial Projects', in Proc. 10th International Design Conference - DESIGN 2008, D. Marjanovic (Ed.), FMENA, Zagreb, p. 1227-1234

[11] Hubka, V. (1982) Principles of Engineering Design, London: Butterworth Scientific, (translated and edited by W.E. Eder from Hubka, V. (1980) WDK 1 - Allgemeines Vorgehensmodell des Konstruierens (General Procedural Model of Designing), Zürich, Heurista; translated into several other languages: French, M. Wyss (1980) Zürich: Heurista; Italian, U. Pighini (1982) Marsilo ed.; Czech, S. Hosnedl (1995) Zürich: Heurista, and others

[12] Hubka, V., Andreasen, M.M., and- Eder, W.E. (1988) Practical Studies in Systematic Design, London: Butterworths 